\title{
Is insulin resistance associated with light at night in healthy sleep deprived individuals?
}

\author{
M. AlBreiki, B. Middleton and S. Hampton \\ Department of Biochemistry \& Physiology, School of Bioscience \& Medicine, Faculty of Health \& Medical Science. \\ University of Surrey, Guildford, GU2 7XH, UK
}

Disruption of the timing of the master biological clock found in suprachiasmatic nuclei (SCN) is associated with metabolic morbidities such as diabetes and coronary heart disease in night-shift workers ${ }^{(1)}$. Light at night is the major component for disruption of $\mathrm{SCN}$ function, resulting in melatonin suppression ${ }^{(2)}$. Melatonin has been linked to a variety of metabolic processes such as lipid and glucose metabolism. Most of the studies have been conducted in animals with only a few human studies reported. This study aims to investigate the impact of light and/or endogenous melatonin on plasma hormones and metabolites prior to and after a set meal in healthy sleep deprived subjects.

A favourable ethical opinion was obtained from the University Ethics Committee. Seventeen healthy participants, 8 females (22.2 years (SD 2.59) BMI 23.62 kg/m² (SD 2.3)) 9 males (22.8 years (SD 3.5) BMI $23.8 \mathrm{~kg} / \mathrm{m}^{2}$ (SD 2.06)) were randomised to a two way cross over design protocol; dim light condition $(<5$ lux $)$ and bright light condition ( $>5001 \mathrm{ux})$, separated by at least seven days. Each session started at 18:00 $\mathrm{h}$ and finished at 06:00 $\mathrm{h}$ the next day. All participants were sleep deprived and semi-recumbent throughout the session. An isocalorific breakfast was consumed at 08:00 h and lunch was timed to be 10 hours before the evening meal. Each participant consumed an evening meal (1066 Kcal, $38 \mathrm{~g}$ protein, $104 \mathrm{~g} \mathrm{CHO}, 54 \mathrm{~g}$ fat, $7 \mathrm{~g}$ fibre) at an individualised time based on estimated melatonin onset. Plasma and saliva samples were collected at specific time intervals to assess glucose, insulin and melatonin levels. Three factor repeated measures ANOVA, followed by Tukeys post hoc test, paired Student's t-test and total area under the curve (TAUC) were performed.

Salivary melatonin levels were significantly higher in the DL compared to BL condition $(\mathrm{p}=0 \cdot 005)$. Postprandial glucose and insulin levels were significantly greater in the BL compared to DL condition $(p=0.02, p=0 \cdot 001)$ respectively. There were significant effects of time in all 3 measures, but no significant effects of gender were seen. TAUC and Student's t-test confirmed the significant differences obtained from repeated measures ANOVA.
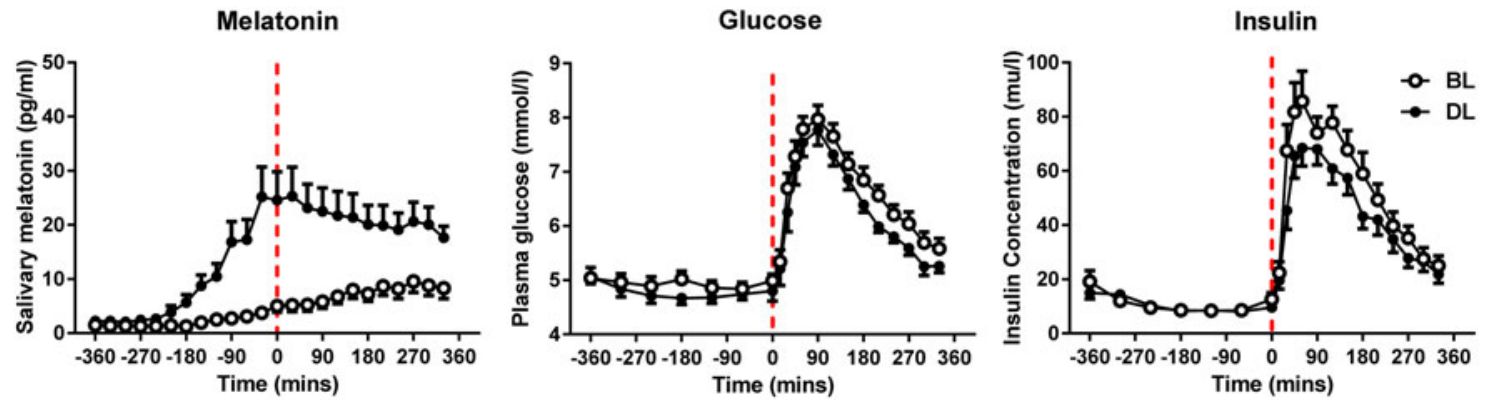

Melatonin suppression was expected due to the light intensity. Postprandial plasma glucose levels were greater in BL than in DL despite the presence of higher insulin levels in the bright light condition these findings could be explained by changes in insulin sensitivity $^{(3,4)}$ These results may have implication for shift-workers.

This study was supported by Abu Dhabi health service company (SEHA).

1. Peschke E. (2008). J Pineal Res 44, 26-40.

2. Zeitzer JM, Dijk DJ, Kronauer R et al. (2000). J Physiol 526, 695-702.

3. DeFronzo RA. (1988). Diabetes 37, 667-687.

4. Scheer F, Hilton MF, Mantzoros CS et al. (2009). PNAS 10, 1-6. 